\title{
Monitoring and managing mothers with gestational diabetes mellitus: a nursing perspective
}

This article was published in the following Dove Press journal:

Nursing: Research and Reviews

20 October 2015

Number of times this article has been viewed

\author{
Diane C Berry' \\ Quinetta B Johnson ${ }^{2,3}$ \\ Alison M Stuebe ${ }^{2,3}$ \\ 'The University of North Carolina \\ School of Nursing, ${ }^{2}$ Women's Primary \\ Health Care, The University of North \\ Carolina School of Medicine, Division \\ of Maternal Fetal Medicine, ${ }^{3}$ The \\ University of North Carolina Gillings \\ School of Global Public Health, \\ Chapel Hill, NC, USA
}

\begin{abstract}
Women diagnosed with gestational diabetes mellitus (GDM) must work in partnership with their health care team to improve both maternal and fetal outcomes. This team may include physicians, midwives, nurse practitioners, physician assistants, registered nurses, certified diabetes educators, and registered dietitians. Management should include medical nutrition therapy, self-monitoring of blood glucose with tight control, and exercise to prevent postprandial hyperglycemia. Approximately $80 \%$ of women diagnosed with GDM are well controlled with medical nutrition therapy, self-monitoring of blood glucose, and exercise; however, approximately $20 \%$ require medication to bring their blood glucose levels under control during pregnancy. The risk of developing type 2 diabetes mellitus decreases dramatically for women who engage in interventions to lose weight postpartum, improve their nutrition and increase their physical activity. Therefore, postpartum women with GDM should be retested and reclassified at 6 weeks postpartum and strongly encouraged to lose weight through proper nutrition and exercise.
\end{abstract}

Keywords: gestational diabetes mellitus, medical nutrition therapy, self-monitoring of blood glucose, exercise, medication, type 2 diabetes

\section{Introduction}

This paper reviews the state of the science on monitoring and managing women diagnosed with gestational diabetes mellitus (GDM) during pregnancy to improve outcomes for both the woman and her fetus. Management of these women includes a combination of medical nutrition therapy (MNT), self-monitoring of blood glucose (SMBG) levels, regular exercise, and medication if needed. In addition, once the infant is delivered, women with GDM need consistent follow-up and a continued balance between nutrition, exercise, and weight loss to prevent progression to type 2 diabetes. This paper focuses on GDM in the current pregnancy, not monitoring of women with previous GDM or preexisting type 2 diabetes or type 1 diabetes. This paper examines diagnosis, prevalence, risk for women and their fetus, and management and monitoring antepartum, and postpartum and long-term follow-up.

\section{Gestational diabetes mellitus}

Approximately a third of women diagnosed with type 2 diabetes have a history of GDM, which has been defined as carbohydrate intolerance ${ }^{1}$ and by the Endocrine Society as hyperglycemia in pregnancy. ${ }^{2}$ The International Association of Diabetes and Pregnancy Study Groups (IADPSG) ${ }^{3}$ and the World Health Organization ${ }^{4}$ define GDM as hyperglycemia first detected during pregnancy. In some cases, hyperglycemia is detected 
for the first time in pregnancy in women with undiagnosed pregestational diabetes; therefore, it is important to screen all women with GDM after delivery to determine whether they meet criteria for diabetes outside of pregnancy.

\section{Pathophysiology}

Insulin resistance develops during the second trimester of pregnancy, and progresses during the third trimester. ${ }^{5}$ The development of insulin resistance is driven by several factors. The placenta secretes hormones and adipokines, including tumor necrosis factor, human placental lactogen, and human placental growth hormone, all of which have been implicated as mediators of insulin resistance in pregnancy. ${ }^{5}$ In addition, overweight, obesity, increased adiposity, increased caloric intake, and decreased exercise all play a part in insulin resistance. ${ }^{5}$ To compensate for the insulin resistance, the pancreas increases maternal insulin production; GDM is diagnosed when maternal insulin production cannot match the demand. ${ }^{5}$

\section{Maternal, fetal, and infant risk}

GDM affects approximately 1 in 20 pregnant women in the United States, ${ }^{1,6}$ and these women are at increased risk of developing GDM in subsequent pregnancies and type 2 diabetes later in life, ${ }^{7-10}$ with a cumulative incidence as high as $70 \% .{ }^{11}$ Risk factors for developing GDM include a family history of diabetes mellitus, prepregnancy overweight or obesity, advanced maternal age, previously large infant, non-White ethnicity, previous unexplained loss or birth of a malformed child, delivering a baby of $\geq 4,000 \mathrm{~g}$, essential hypertension, and pregnancy-related hypertension. ${ }^{6}$ The prevalence of GDM is higher among African Americans, Hispanics/Latinos, Native Americans, and Asians than among non-Hispanic Whites. ${ }^{1}$

Pregestational diabetes is associated with an increased risk of congenital anomalies because of hyperglycemia during organogenesis. ${ }^{6}$ In contrast, women with GDM who have new-onset hyperglycemia in the second or third trimester are not at increased risk of birth defects. ${ }^{6}$ Similarly, women diagnosed with GDM usually do not have the vasculopathy that often accompanies those with pregestational diabetes, so surveillance for retinopathy, nephropathy, and neuropathy is generally not necessary. ${ }^{1}$

Fetal complications include preterm labor and delivery, small or large size for gestational age, shoulder dystocia birth injury, and neonatal hypoglycemia. ${ }^{6}$ Infants born to women with GDM have a twofold risk of developing obesity ${ }^{12}$ and a greater risk of developing type 2 diabetes than infants born to normoglycemic women. ${ }^{13,14}$ Maternal complications include increased risk of hypertension and preeclampsia, and higher rates of cesarean deliveries. ${ }^{6}$

Women with GDM are at increased risk of progression to type 2 diabetes and lifestyle interventions can reduce this risk by $55 \% .{ }^{11}$ The American College of Obstetricians and Gynecologists $(\mathrm{ACOG})^{15}$ and the American Diabetes Association (ADA) ${ }^{1,6}$ therefore recommend that women with GDM be counseled about diet, exercise, and weight reduction. However, several studies suggest that advice is insufficient to change maternal behavior, ${ }^{16-18}$ underscoring the need for comprehensive interventions to reduce long-term maternal disease risk.

\section{Diagnosis}

For decades there has been debate about the criteria for a diagnosis of GDM and whether to use the O'Sullivan and Mahan or Carpenter and Coustan thresholds. ${ }^{19,20}$ Recently, there has also been debate about whether selective or universal screening should be used. ${ }^{15}$ The selective screening approach focuses on women at higher risk, including those at 25 years and older, those less than 25 years of age who are overweight with a body mass index (BMI) of $\geq 25 \mathrm{~kg} / \mathrm{m}^{2}$, those with a family history of diabetes in first-degree relatives, and members of high-risk ethnic or racial group. ${ }^{6} \mathrm{ACOG}$ supports universal screening ${ }^{21}$ and thus screening of all women for GDM is standard between the 24 and 28 weeks of gestation with $50 \mathrm{~g}$ 1-hour oral glucose tolerance test (OGTT) ${ }^{21}$ While there are no proven benefits to early screening for diabetes in early pregnancy, screening can be performed as early as the first prenatal visit if there is a high degree of suspicion that the pregnant woman has undiagnosed type 2 diabetes, a BMI of $\geq 30 \mathrm{~kg} / \mathrm{m}^{2}$, a prior history of GDM or impaired glucose metabolism, or polycystic ovarian syndrome. ${ }^{6,15}$

ACOG recommends using a two-step approach to screening, using a threshold of an abnormal 1-hour screen of $130-140 \mathrm{mg} / \mathrm{dL}$ after a $50 \mathrm{~g}$ glucose challenge and two abnormal values on a 3-hour OGTT after a 100 g glucose challenge and includes a fasting blood glucose. ${ }^{15} \mathrm{GDM}$ is diagnosed if two or more thresholds are met or exceeded on a 3-hour OGTT. ${ }^{15}$ The two-step approach does not require fasting for the first step; however, it does for the second step. This has been a longstanding approach in obstetrical practices; however, the prevalence of GDM with this approach is only approximately $5 \% \cdot{ }^{15}$ In 2011 , ACOG reaffirmed its recommendation of using the two-step approach. 
In 2010, The IADPSG published their revised GDM testing protocol based on the Hyperglycemia and Adverse Pregnancy Outcome Study. ${ }^{22-24}$ The Hyperglycemia and Adverse Pregnancy Outcome Study clarified the association between levels of maternal glucose with perinatal outcomes. ${ }^{22-24}$ The primary outcomes of the study examined infant birth weight, vaginal delivery or cesarean section, neonatal hypoglycemia, and fetal hyperinsulinemia and found an strong association between maternal glucose and birth weight above the 90th percentile and fetal hyperinsulinemia. ${ }^{23}$ IADPSG recommends a one-step approach to screening, using a threshold 1 abnormal value after a $75 \mathrm{~g}$ glucose challenge. ${ }^{3}$ All patients must be fasting for the one-step approach. The prevalence of GDM with this approach is approximately $15 \%-40 \% .^{3}$ The ADA adopted the IADPSG recommendations in $2011 .{ }^{1}$

There remains much debate on whether to use the one-step approach or two-step approach to screening for GDM. ACOG suggests that practitioners and institutions select a single standard for consistency for their practice (Table 1). ${ }^{25}$ The two-step approach has a much lower diagnostic rate of GDM when compared to the one-step approach. This leaves health care providers with no universal protocol making it impossible to compare different studies. In addition, it is difficult to know the true prevalence of GDM and the risks associated with maternal hyperglycemia, and fetal and infant outcomes.

\section{Management of gestational diabetes mellitus}

Management of GDM includes weight management, MNT, SMBG levels, exercise, and if necessary, medication if glucose targets are not met.

\section{Weight management}

It is imperative that women gain the appropriate amount of weight during pregnancy to prevent excessive pregnancy weight gain and postpartum weight retention. The Institute of Medicine has provided recommendations for weight gain during pregnancy based on weight prior to pregnancy. ${ }^{26}$ Underweight women with a BMI $<18.5 \mathrm{~kg} / \mathrm{m}^{2}$ should gain a total of 28-40 pounds including 1 pound a week in the second and third trimesters. ${ }^{26}$ Normal weight women with a BMI of $18.5-24.9 \mathrm{~kg} / \mathrm{m}^{2}$ are advised to gain a total of $25-35$ pounds and 1 pound a week in the second and third trimesters. ${ }^{26}$ Overweight women with a BMI of $25.0-29.9 \mathrm{~kg} / \mathrm{m}^{2}$ are advised to gain a total of 15-25 pounds and 0.6 pounds a week during the second and third trimesters. ${ }^{26}$ Obese women with a BMI of $>30.0 \mathrm{~kg} / \mathrm{m}^{2}$ are advised to gain a total of 11-20 pounds and no more than 0.5 pounds a week during the second and third trimesters. ${ }^{26}$ Higher gestational weight gain is associated with an increased risk of GDM. ${ }^{27}$

\section{Medical nutrition therapy}

MNT for patients with GDM should focus on providing necessary nutrients, normalizing blood glucose levels, preventing ketoacidosis, and supporting appropriate weight gain. ${ }^{28} \mathrm{MNT}$ is the cornerstone of GDM self-management. Every women diagnosed with GDM should be referred to a registered dietitian or a certified diabetes educator as soon as possible and should have regular follow-up visits throughout their pregnancy. The MNT plan should be individualized and based on maternal BMI and focus on macronutrients and micronutrients necessary for maternal and fetal health, normoglycemia, prevention of ketosis, and appropriate maternal weight gain. ${ }^{28}$

Table I Diagnostic criteria for diagnosis of gestational diabetes mellitus

\begin{tabular}{|c|c|c|c|c|}
\hline \multirow{2}{*}{ 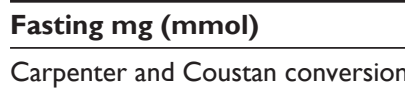 } & \multirow[t]{2}{*}{ I Hour mg (mmol) } & \multicolumn{2}{|c|}{2 Hours $\mathrm{mg}(\mathrm{mmol})$} & \multirow[t]{2}{*}{3 Hours $\mathrm{mg}(\mathrm{mmol})$} \\
\hline & & & & \\
\hline Plasma or serum glucose levels ${ }^{20}$ & $95(5.3)$ & $180(10.0)$ & $155(8.6)$ & $140(7.8)$ \\
\hline \multicolumn{5}{|c|}{ Two-step approach endorsed by ACOG ${ }^{15}$} \\
\hline \multicolumn{5}{|c|}{$50 \mathrm{~g}$ load non-fasting at I hour I30-140 (7.2-7.78) } \\
\hline \multicolumn{5}{|c|}{ If I hour exceeds cutoff, schedule fasting 3-hour OGTT with a $100 \mathrm{~g}$ load } \\
\hline \multicolumn{5}{|c|}{ If fasting glucose is above 126 (6.9), do not proceed } \\
\hline \multicolumn{5}{|c|}{ If fasting glucose is below I 26 (6.9), then proceed with a $100 \mathrm{~g}$ load } \\
\hline \multicolumn{5}{|c|}{ Diagnosis of GDM if two abnormal values on a 3-hour OGTT } \\
\hline Plasma or serum glucose levels & $95(5.3)$ & $180(10.0)$ & $155(8.6)$ & $140(7.8)$ \\
\hline \multicolumn{5}{|c|}{ One-step approach endorsed by IADPSG ${ }^{3}$} \\
\hline \multicolumn{5}{|l|}{$75 \mathrm{~g}$ load fasting 2-hour OGTT } \\
\hline \multicolumn{5}{|c|}{ Diagnosis of GDM if one abnormal value } \\
\hline & $92(5.1)$ & $180(10.0)$ & $153(8.5)$ & \\
\hline
\end{tabular}

Abbreviations: ACOG, American College of Obstetricians and Gynecologists; GDM, gestational diabetes mellitus; IADPSG, International Association of Diabetes and Pregnancy Study Groups; OGTT, oral glucose tolerance test. 
The major goal of MNT in women with GDM is the maintenance of normoglycemia to prevent fetal compromise. ${ }^{28}$ Normoglycemia is defined as a fasting blood glucose of $\leq 95 \mathrm{mg} / \mathrm{dL}(\leq 5.3 \mathrm{mmol} / \mathrm{L})$, a 1 -hour postprandial blood glucose of $\leq 140 \mathrm{mg} / \mathrm{dL}(\leq 7.8 \mathrm{mmol} / \mathrm{L})$, and a 2-hour postprandial blood glucose of $\leq 120 \mathrm{mg} / \mathrm{dL}$ $(\leq 6.7 \mathrm{mmol} / \mathrm{L})^{6,28}$

Many factors affect blood glucose levels, including the amount and types of carbohydrates eaten, exercise, the time of day, and stress. ${ }^{6,28}$ Blood glucose levels and particularly postprandial glucose levels are driven by the amount and types of carbohydrates eaten. ${ }^{6,28}$ Complex carbohydrates such as whole grain bread, whole grain pasta, and brown rice are more slowly absorbed than simple carbohydrates such as sugared sweetened beverages (soda and juice), cakes, cookies, and candy, which are absorbed quicker resulting in a rapid rise of postprandial blood glucose. ${ }^{6,28}$ In women with GDM, the minimum amount of carbohydrates recommended is $175 \mathrm{~g}$ /day, divided between three meals and three snacks to assist women in reaching normoglycemia. ${ }^{6,28}$ Caloric distribution should be $40 \%$ from carbohydrates, $20 \%$ from protein, and $40 \%$ from fat. ${ }^{6,28}$ Keeping postprandial blood glucose levels within the goals set help to decrease the incidence of fetal macrosomia. ${ }^{6}$

To promote normoglycemia and avoid excessive weight gain, dietary guidelines should be basic and easy to understand and implement for women with GDM. ${ }^{6,26,28}$ An individualized meal plan should be reviewed with each woman, taking into account dietary and cultural preferences. ${ }^{6,28}$ General suggestions include the following: women should eat three small, consistent meals and three small snacks per day. ${ }^{6,28}$ Eating smaller meals decreases the risk of postprandial hyperglycemia and preprandial ketosis. ${ }^{6,28}$ Snacks help to prevent overeating at the next meal, and when protein is added to a snack, there is a lower glycemic response. ${ }^{6,28}$ Because many women with GDM have high morning blood glucose levels, they should be encouraged to eat a small breakfast that is low in carbohydrates $(<10 \%)$ and high in protein and fiber, to decrease postprandial hyperglycemia. ${ }^{6,28}$ They should eat low-calorie free foods throughout the day, including lettuce, celery, broccoli, cauliflower, and tomatoes, ${ }^{6,28}$ and avoid highly processed foods, which are often high calorie, high fat, contribute to excessive weight gain, and rapidly increase postprandial blood glucose levels. ${ }^{6,28}$ Most importantly, women should avoid concentrated sweets, which are high in calories and also cause a rapid rise in postprandial glucose levels. ${ }^{6,28}$ Approximately $80 \%$ of women are able to maintain normoglycemia with dietary changes; however, $20 \%$ may require insulin. ${ }^{6}$
Women with GDM should develop a meal plan they can maintain in partnership with their provider. Providers should encourage three small meals and three small snacks per day and discuss with women the composition of the diet (carbohydrates, protein, and fat), portion size, and appropriate calorie level. ${ }^{28}$ The number of servings from each food group should be reviewed and a sample menu should be created.$^{28}$ To ensure that the patient understands how to create a menu, ask her to create a sample menu using resources such as "Choose Your Foods", "Plan Your Meals", or "Exchange Lists for Meal Planning", which are available for free on the Internet.

To date, there is no validated method to estimate energy requirements of women who are overweight or obese. Providers may use the following guidelines to calculate daily nutritional intake for women with GDM. If a woman is less than $80 \%$ of ideal body weight, then the woman should be advised to take in $40 \mathrm{kcal}$ per present pregnant weight in kilograms ${ }^{6,28}$ If a woman is $80 \%-120 \%$ of ideal body weight, then she should aim for $30 \mathrm{kcal}$ per present pregnant weight in kilograms. ${ }^{6,28}$ If she is $120 \%-150 \%$ of ideal body weight, then she should be advised to take in $24 \mathrm{kcal}$ per present pregnant weight in kilograms. ${ }^{6,28}$ If she is $>150 \%$ of ideal body weight, then she should take in $12-15 \mathrm{kcal}$ per present pregnant weight. ${ }^{6,28}$

Some providers use a nutritional assessment, weight history, clinical judgment and previous experience to estimate caloric requirements. Whatever method the provider uses, the focus should be on nutritional adequacy for mother and fetus, weight gain pattern, and blood glucose pattern.

\section{Self-monitoring blood glucose}

Women should keep records of SMBG and a food diary and bring these to each provider visit. The SMBG records and food diary should include the time of the SMBG test and the time, composition, and amount of food and beverages that are consumed. ${ }^{6}$ Together the woman and the provider should examine the records to identify food sensitivities that produce a high glycemic response. ${ }^{28}$ In addition, this review allows the provider to evaluate the woman's understanding of the meal plan and provides the woman with feedback on how much to eat and drink, empowering the woman to take control of her GDM and MNT. ${ }^{28}$

SMBG is measured to demonstrate glycemic control and provide the woman with feedback on her self-management. The woman should be asked to check fasting blood glucose upon rising in the morning, and 1-2 hours after 
breakfast, lunch, and dinner. The MNT prescription only works if there is little to no postprandial hyperglycemia. Two criteria should be met to assure that glycemic control is adequate to prevent macrosomia. First, the fasting blood glucose should be less than $95 \mathrm{mg} / \mathrm{dL}(<5.3 \mathrm{mmol} / \mathrm{L})$, less than $140 \mathrm{mg} / \mathrm{dL}(7.8 \mathrm{mmol} / \mathrm{L})$ at 1 -hour postprandial, and less than $120 \mathrm{mg} / \mathrm{dL}(6.7 \mathrm{mmol} / \mathrm{L}) 2$-hour postprandial. ${ }^{15}$ If these criteria cannot be met after 2 weeks of dietary implementation or they can be met only with severe calorie restriction and weight loss, then medication should be started. ${ }^{6}$ Daily SMBG has been the standard for women with GDM; however, new research has shown that SMBG testing every other day or every 3rd day would not lead to significant delays in the initiation of medication therapy in women with mild GDM, defined as a fasting plasma glucose of $<95 \mathrm{mg} / \mathrm{dL} .{ }^{29} \mathrm{~A} 1 \mathrm{C}$ levels are not routinely used to screen for GDM, but have been found useful to assess glycemic control during pregnancy. ${ }^{6}$

\section{Exercise}

Exercise is an important part of glucose control and decreases blood glucose levels by increasing glucose transfer into the cells without insulin. ${ }^{30}$ Women should therefore be informed of the importance of gentle exercise throughout pregnancy. The ACOG recommends 30 minutes of exercise on most if not all days of the week. ${ }^{31}$ An exercise program for a pregnant woman, however, should take into consideration the woman's prepregnancy exercise routine and build on that. ${ }^{31}$ If a woman was partaking in moderate exercise before pregnancy, then she can be encouraged to continue to her level of comfort. ${ }^{31}$ Appropriate exercise could include walking and stationary bicycling. ${ }^{31}$ Women should be encouraged to discuss their exercise level with their provider. They should be taught to count their heart rate and given a range that is healthy for them and their fetus. In addition, they should be taught to evaluate themselves for uterine contractions and to stop exercising immediately if they occur. ${ }^{31}$ Whatever is decided upon must be safe for both the mother and the fetus and must not lead to fetal distress, uterine contractions, or maternal hypertension (blood pressure $>140 / 90 \mathrm{~mm} / \mathrm{Hg}) .{ }^{31}$ Women should understand that contraindications to exercise include vaginal bleeding, placenta previa, cardiac disease, severe hypertension, malpresentation, intrauterine growth retardation, and morbid obesity. ${ }^{31}$ Pregnant women should not engage in contact sports such as soccer, football, or hockey, or in sports such as rock climbing, gymnastics, or downhill skiing, which have a high potential for falling. ${ }^{15,32}$

\section{Medication management}

If MNT and exercise are not successful in bringing blood glucose levels to below $95 \mathrm{mg} / \mathrm{dL}$ ( $<5.3 \mathrm{mmol} / \mathrm{L}$ ) fasting, below $140 \mathrm{mg} / \mathrm{dL}$ (7.8 mmol/L) 1-hour postprandial, and below $120 \mathrm{mg} / \mathrm{dL}$ (6.7 mmol/L) 2-hour postprandial, then medication should be initiated. ${ }^{6}$ Approximately $20 \%$ of women with GDM require medication during their pregnancy. On the basis of evidence of equivalent efficacy, ACOG considers both insulin and oral medications to be the first-line therapy for GDM. Careful balancing of medication and meals must be instituted to prevent maternal hypoglycemia. ${ }^{33}$

Insulin has historically been the medication of choice in women with GDM, because the majority of insulin preparations have been determined not to cross the placenta. ${ }^{34}$ Before initiating insulin therapy in women with GDM, the woman should receive education on the basic timing and action of insulin, proper injection technique with practice, the need for dietary adjustments, and recognition, avoidance, and treatment of hypoglycemia. ${ }^{34}$ Each woman's insulin regime should be calculated based on her personal needs. ${ }^{6,34}$ Human neutral protamine Hagedorn insulin is the most common insulin used as a part of a basal/bolus regime in women with GDM, and is considered intermediate acting. ${ }^{34}$ Neutral protamine Hagedorn insulin has an onset of 1-3 hours, peaks in 8 hours and has a duration of 12-16 hours. ${ }^{34}$

Human analog insulin is considered the least immunogenic of all insulin preparations. ${ }^{34}$ Lispro (Humalog) and aspart (NovoLog) are rapid onset insulin analogs and are used as bolus (mealtime) insulin. ${ }^{34}$ They have a 15 minutes onset, peak in 30-90 minutes and have a duration of 3-5 hours. ${ }^{34}$ Lispro and aspart have been investigated in pregnant women and are classified as Category B and considered safe with minimal transport across the placenta and have been found to decrease postprandial hyperglycemia and have a lower risk of postprandial hypoglycemia compared to regular insulin preparations. ${ }^{34}$ Long acting (basal) insulin such as detemir (Levemir) and glargine (Lantus) have a 60 minutes onset with no peak and a duration of 20-26 hours. ${ }^{34}$ Detemir is considered a Category $\mathrm{B}$ drug and glargine is a Category $\mathrm{C}$ drug in pregnancy that has been found unlikely to cross the placenta. ${ }^{34,35}$ The most common oral antihyperglycemic medications used in pregnancy include the insulin sensitizers and insulin secretagogues. ${ }^{34}$ The most common insulin sensitizer used in pregnancy is the biguanide metformin. ${ }^{34}$ Biguanides stimulate glucose intake in the liver and periphery and suppress hepatic glucose output. ${ }^{34}$ Metformin (Glucophage) is Pregnancy Class B and has been found to cross the placenta, with cord levels twice maternal levels. ${ }^{36}$ When metformin 
and insulin were examined by Rowan et $\mathrm{al}^{37}$ in a randomized clinical trial (MiG Trial), metformin alone or with supplemental insulin was not associated with increased perinatal complications. Although women randomized to metformin seemed to prefer this mode of medication delivery, unfortunately, approximately 50\% still required insulin to meet their glycemic goals. ${ }^{37}$ Further well-designed randomized controlled trials are needed to fully examine the risk to the fetus. Patients who decide to take metformin should be counseled on the unknown risks to the fetus.

The most common insulin secretagogues used in pregnancy include sulfonylureas. ${ }^{34}$ The mode of action of sulfonylureas is through binding of sulfonylurea receptors in the $\beta$-cells in the pancreas, which in turn stimulate insulin secretion. ${ }^{34}$ Sulfonylureas act by suppressing hepatic glucose production and improving insulin secretion after meals. ${ }^{34}$ First-generation sulfonylureas include tolbutamide, chlorpropamide, and tolazamide. ${ }^{34}$ Second-generation sulfonylureas include glipizide (Glucotrol), glyburide (Diabeta, Micronase), and glimepiride (Amaryl). ${ }^{34}$ In general, sulfonylureas lower glucose by approximately $20 \%$ and hypoglycemia is a serious side effect. ${ }^{34}$ The preferred insulin secretagogue to use in pregnancy is glyburide because it crosses the placenta at a very low rate and benefits the mother directly and fetus indirectly. ${ }^{34}$ Langer et al ${ }^{38}$ compared insulin and glyburide in women with gestational diabetes and found that glyburide was a clinically acceptable alternative to insulin. Of note, one study demonstrated that cord blood levels were approximately $70 \%$ of maternal levels. ${ }^{33}$ To date, no longitudinal studies have examined adverse effects on infants.

\section{Postpartum management}

Approximately two-thirds of mothers with GDM will have GDM in a subsequent pregnancy. ${ }^{6}$ The women most affected are older, more parous, and have greater weight gain between pregnancies. ${ }^{6}$ Risk factors for future impaired glucose tolerance (IGT) and type 2 diabetes include higher gestational insulin requirements, obesity, elevated fasting glucose levels during pregnancy, and early gestational age at the time of diagnosis. ${ }^{6}$ A 5 -year incidence of type 2 diabetes mellitus has been found in $50 \%$ of women with a history of GDM. ${ }^{6}$ Furthermore, while the majority of women with GDM who develop diabetes after pregnancy develop type 2 diabetes, a small subset may develop type 1 diabetes. ${ }^{6}$ The presence of autoantibodies to pancreatic $\beta$-cell antigens is highly predictive of the development of type 1 diabetes. ${ }^{6}$

Among women with GDM, longer duration of breastfeeding is associated with a reduction in maternal risk of type 2 diabetes, ${ }^{39}$ greater insulin sensitivity, ${ }^{40}$ and reduced risk of developing metabolic syndrome. ${ }^{41}$ However, there is also evidence suggesting that more severe maternal glucose intolerance is associated with delayed onset of lactogenesis. ${ }^{42,43}$ Thus, women with GDM may need extra support to initiate and sustain breastfeeding.

After the birth of their infant, women should have their blood glucose checked during the postpartum period to ensure that they return to normal. ${ }^{6}$ If a woman's postpartum fasting blood glucose in the postpartum period remains $>200 \mathrm{mg} / \mathrm{dL}$ then many providers will start the patient on metformin prior to discharge from the hospital. At 6 weeks postpartum, women should have a 2-hour OGTT and should be reclassified as normoglycemia, IGT or diabetes mellitus, according to ADA guidelines. ${ }^{6}$ For women with impaired fasting glucose, IGT or both, MNT, weight loss, and physical activity counseling are recommended, along with the annual assessment of glycemic status. ${ }^{6}$ Initiation of metformin may also be considered. For women with normal glucose testing at 6 weeks, weight loss and physical activity counseling are recommended, with the assessment of glycemic status every 3 years. ${ }^{15}$

\section{Conclusion}

Women diagnosed with GDM must work in partnership with their health care providers to improve both maternal and fetal outcomes. The health care team should work with the woman to focus on MNT, SMBG with tight control, exercise, and medication if necessary to decrease fasting and postprandial hyperglycemia. Women should be encouraged to engage in interventions to lose weight postpartum, improve their nutrition, and increase their physical activity to prevent progression to type 2 diabetes. ${ }^{6}$ Therefore, postpartum women with GDM should be retested and reclassified at 6 weeks postpartum and strongly encouraged to lose weight through proper nutrition and exercise.

\section{Disclosure}

The authors report no conflicts of interest in this work.

\section{References}

1. American Diabetes Association. Standards of Medical Care in Diabetes2015. Diabetes Care. 2015;38:S1-S93.

2. Blumer I, Hadar E, Haden DR, et al. Diabetes in pregnancy: an endocrine society clinical practice guideline. J Clin Endocrinol Metab. 2013;98: 4227-4249.

3. International Association of Diabetes and Pregnancy Study Group Consensus Panel. International association of diabetes and pregnancy study groups recommendations on the diagnosis of hyperglycemia in pregnancy. Diabetes Care. 2010;33:676-682.

4. World Health Organization. Diagnostic Criteria and Classification of Hyperglycemia First Detected in Pregnancy. Geneva: World Health Organization; 2013. 
5. Cunningham F, Leneno K. Williams Obstetrics. 24th ed. New York: McGraw-Hill Education; 2014.

6. American Diabetes Association. Medical Management of Pregnancy Complicated by Diabetes. 5th ed. Alexandria, Virginia: American Diabetes Association; 2013.

7. Bottalico JN. Recurrent gestational diabetes: risk factors, diagnosis, management, and implications. Semin Perinatol. 2007;31:176-184.

8. Kim C, Newton KM, Knopp R. Gestational diabetes and the incidence of type 2 diabetes. Diabetes Care. 2002;25:1862-1868.

9. American Diabetes Association. Standards of medical care in diabetes. Diabetes Care. 2010;33:S11-S61.

10. Albareda M, Caballero A, Badell G, et al. Metabolic syndrome at follow-up in women with and without gestational diabetes mellitus in index pregnancy. Metabolism. 2005;54:1115-1121.

11. Ratner RE. Prevention of type 2 diabetes in women with previous gestational diabetes. Diabetes Care. 2007;30(S242-S245).

12. Reese EA, Leguizamon G, Wiznitzer A. Gestational diabetes; the need for a common ground. Lancet. 2009;373:1789-1797.

13. Clausen TD, Mathiesen ER, Hansen T, et al. High prevalence of type 2 diabetes and pre-diabetes in adult offspring of women with gestational diabetes mellitus or type 1 diabetes: the role of intrauterine hyperglycemia. Diabetes Care. 2008;31:340-346.

14. Yoger Y, Visser GH. Obesity, gestational diabetes and pregnancy outcome. Semin Fetal Neonatal Med. 2009;14:77-84.

15. American College of Obstetricians and Gynecologists. Gestational diabetes mellitus. Washington, DC: American College of Obstetrics and Gynecologists; 2013.

16. Polley BA, Wing RR, Sims CJ. Randomized controlled trial to prevent excessive weight gain in pregnant women. Int $J$ Obes Relat Metab Disord. 2002;26:1494-1502.

17. Leermakers EA, Anglin K, Wing RR. Reducing postpartum weight retention through a correspondence intervention. I. Int J Obes Relat Metab Disord. 1998;22:1103-1109.

18. O'Toole ML, Sawicki MA, Artal R. Structured diet and physical activity prevent postpartum weight retention. $J$ Womens Health. 2003;12(10):991-998.

19. O'Sullivan GB. Gestational diabetes. Unsuspected, asymptomatic diabetes in pregnancy. $N$ Eng J Med. 1961;264:1082-1085.

20. Carpenter MW, Coustan DR. Criteria for screening tests for gestational diabetes Am J Obstet Gynecol. 1982;144:768-773.

21. American College of Obstetricians and Gynecologist Committee on Practice Guidelines-Obstetrics. ACOG Practice Bulletin Number 30: Gestational Diabetes. Obstet Gynecol. 2001;98:525-538.

22. HAPO Study Cooperative Research Group. The Hyperglycemia and Adverse Pregnancy Outcome (HAPO) Study. Int J Gynaecol Obstet. 2002;78:69-77.

23. HAPO Study Cooperative Research Group. Hyperglycemia and Adverse Pregnancy Outcome (HAPO) Study. NEng J Med. 2008;358:1991-2002.

24. HAPO Study Cooperative Research Group. Hyperglycemia and Adverse Pregnancy Outcome (HAPO) Study: associations with neonatal anthropometrics. Diabetes. 2009;58:453-459.

25. Berggren EK, Boggess KA, Stuebe AM, Jonsson Funk M. National Diabetes Data Group vs Carpenter-Coustan criteria to diagnose gestational diabetes. Am J Obestet Gynecol. 2011;205(3):e1-e7.

26. Institute of Medicine. Weight Gain During Pregnancy: Reexamining the Guidelines. Washington, DC: National Academies Press; 2009.
27. Morisset AS, St-Yves A, Veillette J, Weisnagel SJ, Tchernof A, Robitaille J. Prevention of gestational diabetes mellitus: a review of studies on weight management. Diabetes Metab Res Rev. 2010;26(1):17-25.

28. Franz MJ, Evert A. American Diabetes Association Guide to Nutrition Therapy for Diabetes. 2nd ed. Alexandria, VA: American Diabetes Association; 2012.

29. Mendez-Figueroa H, Daley J, Lopes VV, Coustan DR. Comparing daily versus less frequent blood glucose monitoring in patients with mild gestational diabetes. J Matern Fetal Neonatal Med. 2013;26(13): $1268-1272$.

30. Petry C. Gestational diabetes:origins, complications, and treatment. Boca Raton, FL: CRC Press; 2014.

31. The American College of Obstetricians and Gynecologists. Exercise during pregnancy and the postpartum period: ACOG Committee Opinion Number 267. Washington, DC: The American College of Obstetricians and Gynecologists; 2002.

32. Coustan DR. Gestational diabetes mellitus. Clin Chem. 2013;59(9): $1310-1321$.

33. Hebert MF, Ma X, Naraharisetti SB, Krudys KMea. Are we optimizing gestational diabetes treatment with glyburide?: the pharmacologic basis for better clinical practice. Clin Pharmacol Ther. 2009;85:607-614.

34. Physicians' Desk Reference Staff. Physicians'Desk Reference. 69th ed. Montvale, NJ: PDR Network; 2015.

35. Pollex EK, Feig DS, Lubetsky A, Yip PM, Koren G. Insulin glargine safety in pregnancy: a transplacental transfer study. Diabetes Care. 2010;33:29-33.

36. Vanky E, Zahlsen K, Spigset O, Carlsen SM. Placental passage of metformin in women with polycystic ovary syndrome. Fertil Steril. 2005;83:1575-1578.

37. Rowan JA, Hague WM, Gao W, Battin MR, Moore MP; MiG Trial Investigators. Metformin versus insulin for the treatment of gestational diabetes. N EnglJ Med. 2008;358:2003-2015.

38. Langer O, Conway DL, Berkus MD, Xenakis EM, Gonzales O. A comparison of glyburide and insulin in women with geatational diabetes. N Eng J Med. 2000;343(16):1134-1138.

39. Ziegler AG, Wallner M, Kaiser I, et al. Long-term protective effect of lactation on the development of type 2 diabetes in women with recent gestational diabetes mellitus. Diabetes. 2012;61(12):3167-3171.

40. Chouinard-Castonguay S, Weisnagel SJ, Tchernof A, Robitaille J. Relationship between lactation duration and insulin and glucose response among women with prior gestational diabetes. Eur J Endocrinol. 2013;168(4):515-523.

41. Gunderson EP, Jacobs DR Jr, Chiang V, et al. Duration of lactation and incidence of the metabolic syndrome in women of reproductive age according to gestational diabetes mellitus status: a 20-Year prospective study in CARDIA (Coronary Artery Risk Development in Young Adults). Diabetes. 2010;59(2):495-504.

42. Matias SL, Dewey KG, Quesenberry CP Jr, Gunderson EP. Maternal prepregnancy obesity and insulin treatment during pregnancy are independently associated with delayed lactogenesis in women with recent gestational diabetes mellitus. Amer J Clin Nutr. 2014;99(1):115-121.

43. Nommsen-Rivers LA, Dolan LM, Huang B. Timing of stage II lactogenesis is predicted by antenatal metabolic health in a cohort of primiparas. Breastfeeding Med. 2012;7(1):43-49.

resource management, health outcomes, and improving patient safety in all settings. The manuscript management system is completely online and includes a very quick and fair peer-review system. Visit http://www.dovepress.com/testimonials.php to read real quotes from published authors.

\section{Dovepress}

Nursing: Research and Reviews

\section{Publish your work in this journal}

Nursing: Research and Reviews is an international, peer-reviewed, open access journal publishing original research, reports, reviews and commentaries on all aspects of nursing and patient care. These include patient education and counselling, ethics, management and organizational issues, diagnostics and prescribing, economics and 